adolescents with average body mass index of 34 plus or minus 3.8 and compared with 50 age- and sex-matched controls, with a body mass index of 21.6 plus or minus 1.9. Cardiac dimensions, stroke volume, left ventricular and right ventricular systolic and diastolic functions were evaluated.

Results The obese group had a higher end-diastolic septal and posterior wall thickness and left ventricular mass index than the nonobese group. Body mass index, mid-arm and hip circumference values showed significant correlations with these echocardiographic variables. Systolic and diastolic functions of the left ventricle were normal in both groups, although stroke volume was high in the obese group. The right ventricle tissue Doppler parameters were similar in both groups. However, the S wave of the septal/lateral tricuspid valve annulus was reduced in the obese group, but not to the level reflecting systolic dysfunction. This was inversely correlated with hip, waist, and mid-arm circumference. Stepwise multiple regression analysis showed that the mid-arm and hip circumferences followed by the body mass index are significant predictors of these early cardiac abnormalities.

Conclusion Left ventricular hypertrophy is present in obese children, although both systolic and diastolic functions are normal. Tissue Doppler imaging revealed a minor, but still significant, reduction in the right ventricular systolic function.

\section{LOW DOSE OXYBUTININ IN CHILDHOOD NOCTURNAL ENURESIS}

doi:10.1136/archdischild-2012-302724.1676

M Naseri. Mashhad University of Medical Sciences, Mashhad, Iran

Objective To evaluate response to low dose oxybutynin in children with nocturnal enuresis.

Materials and Methods Forty one out of 111 neurologically normal enuretic children who referred nephrology clinic in a 3-year period (2007-2009)received low dose oxybutynin (2.5-10 mg/day depending on the age) to define response to the drug in one and 3 month periods. No response, partially and full responses were defined as decreased in bed wetting in the rate of $0-49 \%, 50-89 \%$ and $\geq 90 \%$ respectively.

Results In first month of treatment, full, partial and no responses were reported in 3 (7.3\%), 14 (34.1\%) and 24 (58.6\%) patients respectively. In non-responder patients $6(25 \%)$ and 5$) 20.8 \%$ (patients had full and partial responses in 3-month period, whereas $13(54.2 \%)$ had no response. Side effects of the drug were reported in $5(12.2 \%)$ patients. Children with non-mono symptomatic nocturnal enuresis presented a better response to the drug than those with mono symptomatic nocturnal enuresis (75\% versus $25 \%$ ). There was no significant differences between age, gender, family history of enuresis and presence of absence of daytime urinary or bowel symptoms in responder and non-responder groups ( $p>0.05$ for all).

Conclusion In the present study which is a clinical report study with no control group, there was $68.3 \%$ treatment benefit and $12 \%$ risk (side effects of the drug) with low dose oxybutynin, so it may have a role in treating nocturnal enuresis especially patients with NMNE who experience adverse effects of the drug with standard treatment.

\section{MONOSYMPTOMATIC AND NON-MONO SYMPTOMATIC NOCTURNAL ENURESIS: A CLINICAL EVALUATION}

doi:10.1136/archdischild-2012-302724.1677

'M Naseri, ${ }^{2} \mathrm{M}$ Hiradfar. 'Mashhad University of Medical Sciences; ${ }^{2}$ Pediatric Surgery, Mashhad University of Medical Sciences, Mashhad, Iran

Background Nocturnal enuresis is divided into mono-symptomatic nocturnal enuresis) MNE) and non mono-symptomatic nocturnal enuresis) NMNE. (This study was conducted to review clinical and ultrasonography findings in enuretic children, and compare organic and functional pathologies of lower urinary tract (LUT) in children with mono-MNE with those who have NMNE.

Methods 111 neurologically normal children with chief complaint of enuresis enrolled in the study including 60 boys and 51 girls, aged 5-17 years old, 43 (38.8) \% with MNE and 68 (61.2) \% with NMNE. Urine analysis, urine culture and kidney-bladder ultra sonography was done for all. Some patients underwent voiding cystoureterography (VCUG), urodynamic study (UDS), or both.

Results Patients were divided in to 3 groups: MNE, NMNE -daytime incontinence and NMNE+daytime incontinence. Constipation, encopresis and urge incontinence were significantly more frequent in patients with NMNE+daytime incontinence $(p=0.011$, $0.003,0.001$ respectively). Bladder wall thickness was the most common US findings. One patient with MNE and 9 with NMNE+ daytime incontinence had vesico-ureteral reflux (VUR) ( $p=0.016)$. Posterior urethral valve was reported in one patient with NMNE. Evidences of bladder dysfunction were noted in about half of the patients who underwent UDS, with higher prevalence in cases with NMNE+daytime urinary incontinence $(p=0.297)$. Bowel symptoms and VUR were significantly more prevalent in cases with NMNE +daytime incontinence.

Conclusion We recommend doing VCUG in enuretic children who have daytime incontinence. In addition our study revealed that symptoms suggestive of over active bladder are not good indicators for bladder dysfunction.

\section{TREATMENT OF INFANTILE COLIC WITH LACTOBACILLUS REUTERI AND THE RELATIONSHIP WITH POSTPARTUM DEPRESSION: A RANDOMIZED CONTROLLED TRIAL STUDY}

doi:10.1136/archdischild-2012-302724.1678

N Karadag, D Dilli, A Dursun, B Karagol, N Hakan, A Kundak, S Beken, A Zenciroglu, N Okumus. Dr Sami Ulus Maternity, Childrens Education and Research Hospital, Division of Neonatology, Ankara, Turkey

Background and Aim Infantile colic (IC) is a common behavioural syndrome within the first 3 months of life. We aimed to compare the effectiveness of Lactobacillus reuteri (L.reuteri) and herbal drop combined with baby massage on IC and to evaluate the relationship with postpartum depression and anxiety.

Methods Wessel Criteria were used for the diagnosis of IC in otherwise healty newborns. Patients were randomized into three groups: Group-1: L. Reuteri DSM17938 (BioGaia AB, Stockholm, Sweden), Group-2: Herbal drop (Babs, BerkoAB, İstanbul, Turkey), Group-3: Sterile water. All groups had baby massage. Infants crying episodes, feeding and stooling characteristics, growth, weight gain were noted weekly. Emotional structures of mother's behaviour were evaluated before and at 2nd month with Edinburgh postpartum depression scale (EPDS) and State Trait Anxiety Inventory (STAI) tests.

Results Seventy-four infants and their mothers completed the trial: 25 in the probiotic group, 24 in herbal drop, and 25 in placebo group. There was no difference between groups in demographic and clinical characteristics. The male rate was $62.2 \%(n=46)$. Crying times(minutes/day) at admission were similar (Group1: 348 \pm 37 , Group2: $353 \pm 30$, Group3: $346 \pm 28, p=0.67)$. A significant decrease in crying times were seen at 3.week in group1 (Group1: 35 \pm 9.8 , Group2: $300 \pm 32.2$, Group3: $188 \pm 40, p=0.001)$. The weight gain at this time was in group1 better, too $(p=0.001)$. High EPDS and STAI scores decreased significantly after treatment in group 1 at 2 month $(p=0.001)$. No adverse effects were reported in our groups.

Conclusion The emotional structure and anxiety level of mothers affects the behaviour of infants. L. reuteri, reduced the crying episodes, mothers anxiety and postpartum depression and can be used in treatment of infantile colic. 
0 Maiga, B Reime, A Hahn, B Kreuels, W Loag, J May. Infectious Disease Epidemiology, Bernhard-Nocht-Institute for Tropical Medicine, Hamburg, Germany

Background and Aims In Ghana, 74/1,000 children per year die before their fifth birthday and many suffer from ill health. We explore the relative impact of genetic traits for the children's health. Methods Within a trial on Intermittent Preventive Treatment in Infants for malaria 1,070 infants were recruited at 3 months and followed-up for 21 months in the Ashanti region. Each month standardized medical history, clinical exam and parasite density were taken. Anthropometric measures were performed every 3 months. DNA preparation and genotyping were performed according to standardized protocols. We defined "health" as a lack of malaria episodes with high parasitaemia, no episodes of anemia, no reporting of severe events like measles, accidents, burns, pneumonia, and normal WHO-growth standards during the entire study phase. We tested the association between health and genetic traits in multivariable logistic regression analyses adjusted for socioeconomic, spatial and clinical data.

Results As expected, children with sickle cell trait (HbAS) were more likely to grow up healthy $(\mathrm{OR}=2.89,95 \%-\mathrm{CI}=1.59-5.24)$. This effect was less pronounced for carriers of $\mathrm{HbAC}$ (hemoglobin $\mathrm{C}$ ). The CD74 gene SNP rs7709772AG was associated with health $(\mathrm{OR}=8.00,95 \%-\mathrm{CI}=1.76-36.29)$. This gene encodes for a membrane protein which is important for the regulation of immune responses against infectious diseases. $\alpha$-thalassemia was not related to health in multivariate analyses.

Conclusions Independently of socioeconomic and geographic factors, genetic traits that influence the risks for malaria and other infectious diseases may affect children's health in an endemic area. This information can be relevant for the development of treatments.

\section{AN AUDIT OF IMPACTS OF FRENULECTOMY IN BREAST FEEDING}

doi:10.1136/archdischild-2012-302724.1680

'E Matthews, ${ }^{2} \mathrm{M}$ Choudhry. ${ }^{1}$ Community Midwives, Imperial College Healthcare NHS Trust; 2Paediatric Surgery, Chelsea and Westminster Hospital, Imperial College, London, UK

Background and Aims To review impact of frenulectomy on breast feeding success.

Methods Prospective audit of babies undergoing frenulectomy from December 2010 to January 2012. A pre-procedure questionnaire was completed in all cases. Babies were assessed and procedure was carried out in the clinic under complete aseptic conditions. Babies were breastfed immediately after treatment and observed for 30 minutes. No further clinical review was conducted. A post-procedure questionnaire was returned within 3 weeks.

Results 50 babies underwent frenulectomy. Mean age at procedure was 6.6 weeks and range 1.1-22.3 weeks. Procedures were carried out by a single operator without complications. 26 post procedure questionnaires were returned. Pre and post feeding methods and difficulties are shown in the tables below.

Abstract 1680 Table 1 Breast feeding methods pre and post frenulectomy

\begin{tabular}{lcc}
\hline Feeding Method & Pre-frenulectomy - n [\%] & Post-frenulectomy - n [\%] \\
\hline Breast feeding only & $12[46 \%]$ & $16[61 \%]$ \\
Artificial milk only & $1[4 \%]$ & $2[8 \%]$ \\
Expressed breast milk only & $0[0 \%]$ & $0[0 \%]$ \\
Breast feeding combined with & $13[50 \%]$ & $8[31 \%]$ \\
expressed and/or artificial milk & & \\
\hline
\end{tabular}

Abstract 1680 Table 2 Feeding difficulties pre and post frenulectomy

\begin{tabular}{lcc}
\hline Feeding difficulties & Pre-frenulectomy n [\%] & Post-frenulectomy n [\%] \\
\hline None & $3[12 \%]$ & $14[54 \%]$ \\
Sore nipples/breast & $13[50 \%]$ & $5[19 \%]$ \\
Difficulty attaching/maintaining & $16[62 \%]$ & $2[8 \%]$ \\
Prolonged feeding/unsatisfied & $11[42 \%]$ & $9[35 \%]$ \\
Poor weight gain & $5[19 \%]$ & $1[4 \%]$ \\
\hline
\end{tabular}

Conclusions Frenulectomy has a positive impact on breastfeeding success. We recommend early treatment if feeding difficulties are observed combined with tongue tie.

\section{LATE-PRETERM DELIVERY: PSYCHOLOGICAL DISTRESS AND LACTOGENESIS}

doi:10.1136/archdischild-2012-302724.1681

C Gabrieli, R Ciullo, L Sansone, I Gambina, F Cavallin, D Faggian, M Santini, V Zanardo. Padua University School of Medicine, Agna, Italy

Late-preterm infants represent the most rapidly growing group of preterm births. The majority of these infants is treated as term neonates at the regular nursery.

It is noteworthy that late-preterm infants have a lower breastfeeding prevalence than term neonates.

It is not known whether psychological distress affects lactogenesis or the mother-to-infant bonding.

The study's aim is to verify if late-preterm delivery is associated to psychological distress affecting Mother-to-Infant bonding and breastfeeding.

The study was performed at the Departement of Gynaecology and Human. Reproduction Science of Padua University, between June and October 2011.

The study group consisted of 75 mothers: 25 delivered late-preterm with caesarean section and 50 delivered with term vaginal birth.

The third day after birth, four psychological tests were administered to mothers: STAI, EPDS, MIBS, LüscherColorTest.

Baseline levels of prolactin and cortisol were controlled.

ANOVA shows significantly higher levels of trait anxiety and state anxiety in late-preterm infants' mothers: the average trait anxiety is 46.0 (IOR 39.0-50.0) in late-preterm infants' mothers and 40.5 (IOR35.3-44.0) in term infants' mothers ( $\mathrm{p}=0.01$ ); and the average state anxiety is 48.0 (IOR42.0-56.0) in late-preterm infants' mothers and 43.0 (IOR 39.0-48.8) in term infants' mothers ( $p=0.03$ ) respectively. There are significantly higher levels of depression in late-preterm infants' mothers: in EPDS the average is 9 (IOR 8-13) in late-preterm infants' mothers and 6 (IOR 4-9) in term infants' mothers $(p=0.001)$. In MIBS, joy is lower in late-preterm infants' mothers.

In late preterm infants this is associated to reduced exclusive breastfeeding at discharge ( $20 \%$ vs $66 \%$ ).

The LüscherColourTest is comparable for both groups.

Mothers who delivered late-preterm have negative emotional changes, without hormonal changes. The psychological distress is negatively associated to an altered Mother-to-Infant bonding and lactation performance.

\section{NICE (UK) GUIDELINES FOR SEDATION IN CHILDREN: NEED FOR A PRAGMATIC APPROACH?}

doi:10.1136/archdischild-2012-302724.1682

G Erumbala, I Misra, N Chawda. Paediatrics, Milton Keynes General Hospital NHS Foundation Trust, Milton Keynes, UK 